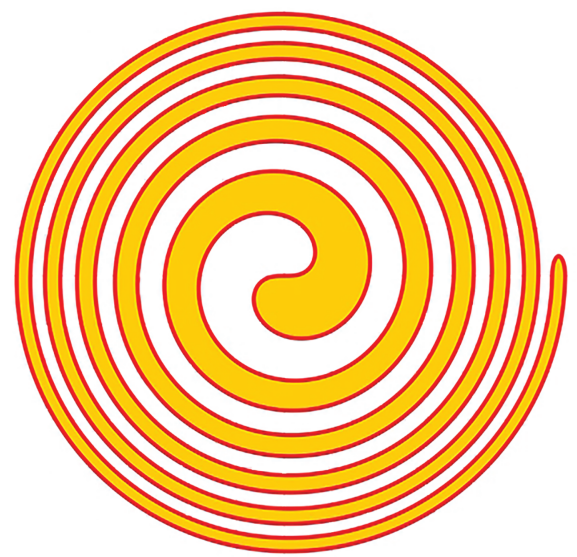

\title{
Ancient Solutions to Geometric Flows
}

\section{Panagiota Daskalopoulos and Natasa Sesum}

A major breakthrough in the history of nonlinear partial differential equations occurred in 2004 with Grigori Yakovlevich Perelman's proof of the Poincaré conjecture and Thurston's geometrization conjecture, which was based on years of work on the Ricci flow by Richard Hamilton.

Thurston's geometrization conjecture, considered to be one of the most important problems in topology, is a generalization of the Poincaré conjecture, stated by Henri Poincaré in 1904. The latter asserts that any closed simply connected three-dimensional manifold is topologically a threedimensional sphere. Simply connected means that any loop on the manifold can be contracted to a point. Analogous results in higher dimensions had been previously resolved by Stephen Smale (in dimensions $n \geq 5$ ) and Michael Freedman (in dimension $n=4$ ), who both received the Fields Medal for their contributions to this problem. The three-dimensional case that Poincaré stated turned out to be the hardest of them all. Of the seven Millennium Prize Problems that were stated by the Clay Mathematics Institute on May 24, 2000, the Poincaré conjecture is the only

Panagiota Daskalopoulos is a professor of mathematics at Columbia University. Her email address is pdaskalo@math. columbia.edu.

Natasa Sesum is a professor of mathematics at Rutgers University. Her email address is natasas@math.rutgers.edu.

Communicated by Notices Associate Editor Chikako Mese.

For permission to reprint this article, please contact:

reprint-permission@ams.org.

DOI: https://doi.org/10.1090/noti2056 one that has thus far been solved, though its proof was envisioned by Richard S. Hamilton in the early 1980s.

The early 1980s was an exciting time for the development of nonlinear elliptic and parabolic PDEs. The fundamental work of N. Krylov and M. Safonov on the Hölder regularity for elliptic and parabolic second-order equations in nondivergence form was opening new fundamental directions for the development of fully nonlinear equations. About the same time, geometric analysts such as R. Hamilton, G. Huisken, R. Schoen, L. Simon, K. Uhlenbeck, and S. T. Yau, among others, were developing new models of nonlinear elliptic and parabolic geometric PDEs, aiming to approach fundamental problems in topology and geometry.

During one of the discussions between Hamilton and Yau the idea of using the Ricci flow for the resolution of the Poincare conjecture was considered. The Ricci flow is the analogue of the heat equation on a Riemannian manifold, evolving a metric defined on this manifold by its Ricci curvature. Heuristically, the Ricci curvature is an intrinsic quantity that measures how much a curved Riemannian manifold deviates from flat Euclidean space in terms of controlling the growth rate of the volume of metric balls in the manifold. The diffusion character of the Ricci flow tends to spread curvature out over the entire manifold. Hence, one expects concentrations of large curvature to be smoothed out, and in the long run the flow may converge to a metric of constant curvature. A similar phenomenon called the smoothing effect is one of the fundamental properties of the heat equation on $\mathbb{R}^{n}$, itself the simplest model 


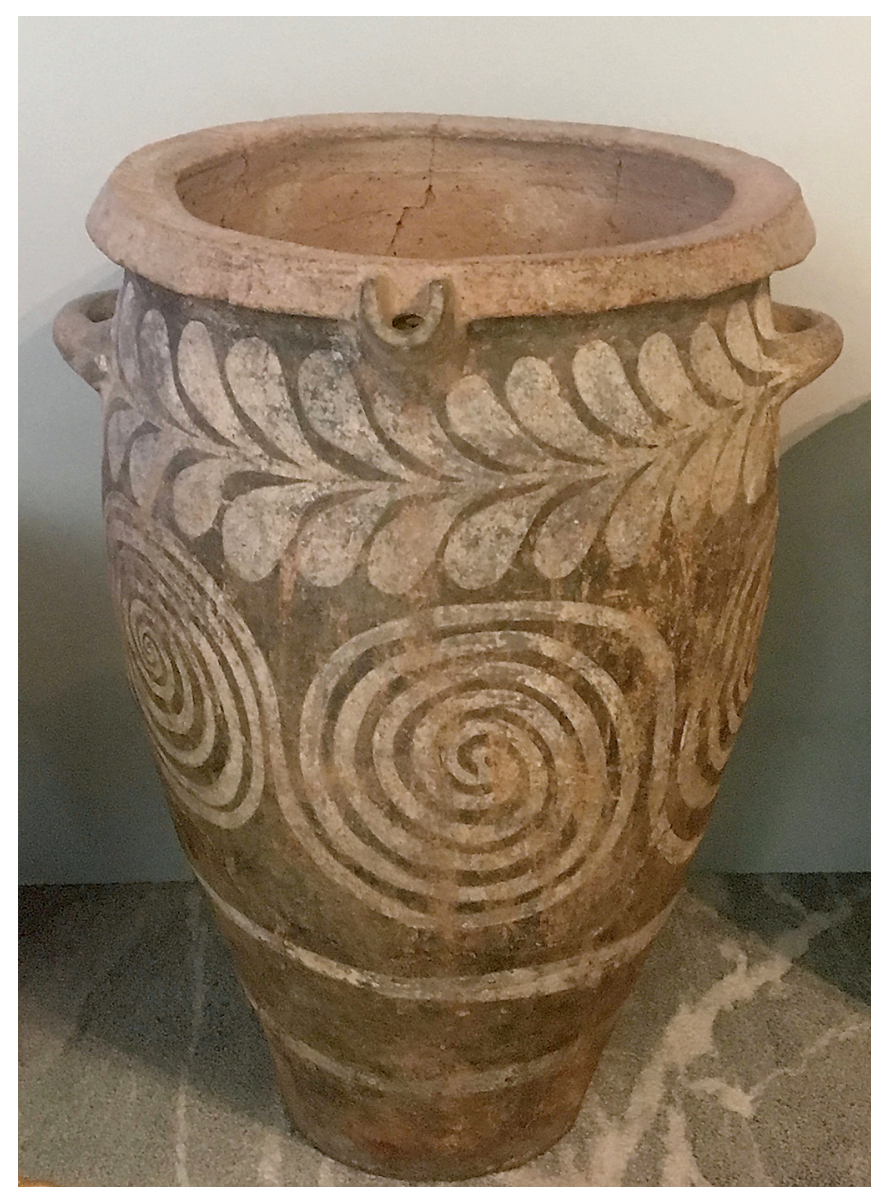

Figure 1. The image on an ancient vase from Minoan Crete dated 1500-2000 BC resembles an ancient solution to curve shortening flow (see Figure 2).

of parabolic partial differential equations describing diffusion of heat in a solid medium. However, the Ricci flow is a nonlinear system of equations, and depending on the initial metric it is likely to develop singularities in finite time. A special approach with new techniques needed to be introduced to carry out Hamilton's idea.

Around the same time, Huisken started studying another important geometric parabolic partial differential equation, the mean curvature flow. This is an example of a geometric flow of hypersurfaces in a Riemannian manifold (e.g., smooth surfaces in three-dimensional Euclidean space). Intuitively, a family of surfaces evolves under mean curvature flow if the normal component of the velocity at which a point on the surface moves is given by the mean curvature of the surface. The mean curvature locally describes the curvature of an embedded surface in some ambient space, the simplest being Euclidean space. For a curve embedded in a plane the mean curvature is just its curvature, as is taught in calculus. It follows easily that the mean curvature flow moves in the direction where the area decreases as fast as possible. It can be understood as an extrinsic version of the heat equation on manifolds. Even though the Ricci flow is an intrinsic flow while the mean curvature flow is an extrinsic flow, they are both nonlinear parabolic equations on manifolds that share many similarities in their behaviors. Hamilton often refers to them as "fraternal twins."

The first results on the Ricci flow [13] and the mean curvature flow [15] marked the beginning of a remarkable development of parabolic equations often referred to as geometric flows. Other important examples of geometric flows are the Gauss curvature flow, a fully nonlinear model of Monge-Ampère type, and the inverse mean curvature flow, another fully nonlinear flow that finds application in general relativity. Despite remarkable recent developments, many important problems remain to be addressed. Later in the article, we will discuss some of these problems that are related to singularities.

In Hamilton's first paper on the Ricci flow, [13], he showed that every closed simply connected threedimensional manifold that admits a metric of positive Ricci curvature is diffeomorphic to the three-sphere. The idea is to start with a Riemannian metric of positive Ricci curvature and deform it by the Ricci flow. He showed that after renormalizing the flow, to keep the volume of the manifold with respect to the evolving metric constant, the flow converges to the round three-sphere. This implies that the initial manifold is diffeomorphic to the three-sphere. Hamilton then wrote a series of papers developing the theory of Ricci flow aiming towards a proof of the geometrization conjecture. Perelman completed Hamilton's program, proving the geometrization conjecture in [18-20].

Briefly, the idea is to begin with any Riemannian metric on a given closed three-manifold and flow it using the Ricci flow equation in order to obtain a metric of constant curvature. As remarked above, this idea worked perfectly in the case when the manifold admits a metric of positive Ricci curvature (see [13]). However, the general case is much more complicated. While Hamilton showed that the Ricci flow always admits a solution for a short time, if the topology of the manifold is sufficiently complicated, then no matter what we choose as an initial metric, the Ricci flow solution will encounter finite-time singularities that may occur along proper subsets of the manifold. Hence, to obtain the conclusion of the Poincare or the geometrization conjecture, one has to do the analysis past the first time a singularity occurs. That is why we need the more general Ricci flow with surgery, which was first introduced by Hamilton in the context of four manifolds. From an analytical point of view, surgery is introduced in order to cut out high curvature regions where singularities occur and replace them with geometrically nice regions. This allows us to restart the Ricci flow with a new metric constructed at a singular time. It is then necessary to show analytical and geometric estimates in order to control both the topology and the 
geometry of the surgery process. Let us remark here that continuing after a singularity occurs is one of the fundamental problems in nonlinear time-dependent PDEs.

Hamilton introduced the Ricci flow with surgery to deal with singular regions as they develop, but he was unable to prove this method "converged" in three dimensions. Perelman completed this in [18-20]. One of Perelman's main contributions was to describe all possible singularities in three dimensions. More precisely, Perelman takes any compact, simply connected, three-dimensional manifold without boundary and starts to run the Ricci flow. This deforms the manifold into round pieces with tubes between them. He cuts the tubes and continues deforming the manifold until he is left with a collection of round three-dimensional spheres. He rebuilds the original manifold by connecting the spheres together with three-dimensional cylinders, showing that the manifold is, in fact, homeomorphic to the sphere.

\section{Singularity Formation and Ancient Solutions}

One of the crucial ingredients in proving the Poincaré and the geometrization conjectures using the Ricci flow is the detailed analysis of singularity formation. This is achieved through dilation arguments around a singularity. In [14], Hamilton proved that if $T_{\max }<\infty$ is the maximal existence time of a smooth solution $\left(M^{n}, g(t)\right)$ to the Ricci flow, then the Riemannian curvature of metric $g(\cdot, t)$ blows up as $t \rightarrow T_{\max }$, namely, $\lim \sup _{t \rightarrow T_{\max }} \sup _{M}|R m|_{g(t)}=\infty$. The Riemannian curvature tensor assigns to each point on a Riemannian manifold a tensor that measures the extent to which the metric tensor is not locally isometric to that of Euclidean space.

Assume that $x_{i} \in M$ and $t_{i} \rightarrow T_{\max }$ are sequences of points and times so that $\lim _{i \rightarrow \infty}\left|\operatorname{Rm}\left(x_{i}, t_{i}\right)\right|=\infty$. If we rescale the metric $g(\cdot, t)$, defining the sequence of solutions

$$
g_{i}(\cdot, t)=K_{i} g\left(\cdot, t_{i}+t K_{i}^{-1}\right),
$$

where $K_{i}=|R m|\left(x_{i}, t_{i}\right)$ and the sequences $x_{i}$ and $t_{i}$ are carefully chosen, we can obtain a smooth solution to the Ricci flow in a limit. Note that we have

$$
-t_{i} K_{i} \leq t<\left(T_{\max }-t_{i}\right) K_{i},
$$

and hence the limiting solution exists for $t \in\left(-\infty, T_{0}\right)$, where $0 \leq T_{0} \leq+\infty$. Such solutions are called ancient solutions. These are special solutions that play an important role in singularity analysis of Ricci flow. Similar blow-up analysis is used to study singularities in other geometric flows as well as for other parabolic equations.

Definition (Ancient solutions). A solution to a parabolic equation is called ancient if for some $T_{0} \leq+\infty$, it is defined for all $t \in\left(-\infty, T_{0}\right)$. In the special case that $T_{0}=+\infty$, the solution is called eternal.
In [18], Perelman made the remarkable discovery that the Ricci flow is a gradient flow of a functional that is monotone along the flow. Using this fact he proved his important $\kappa$-noncollapsing result, which provided a big step forward in the singularity analysis and description of singularities in three-dimensional Ricci flow.

Definition ( $\kappa$-noncollapsed property). A Riemannian metric $g$ is said to be $x$-noncollapsed on the scale $\rho$ if for every $r<\rho$ and every $x \in B(p, r)$ such that $|R m|(x) \leq r^{-2}$, the metric ball $B(p, r)$ has volume at least $\kappa r^{n}$.

Perelman proved that if $M$ is closed and $T<\infty$, then there exists a $\kappa>0$ such that the Ricci flow solution $g_{i j}(t)$ is $x$-noncollapsed. An important corollary of this result is that any complete ancient solution to the Ricci flow that models singularities in the sense described above is $\kappa$-noncollapsed on all scales for some $\kappa>0$. This led Perleman to introduce the following definition.

Definition ( $\kappa$-solution). Any $\kappa$-noncollapsed ancient complete Ricci flow solution with bounded and nonnegative curvature operator is called a $\kappa$-solution.

It is a consequence of known results that every threedimensional complete ancient solution to the Ricci flow has bounded and nonnegative curvature and hence is a $\kappa$ solution. Perelman made the important observation that the singularities of three-dimensional Ricci flow are always modeled on $x$-solutions [19].

On the other hand, in [18] Perelman constructed a rotationally symmetric ancient $x$-noncollapsed solution on $S^{3}$ that is different from the three-sphere, which we will refer to as Perelman's solution. This ancient solution has backward-in-time limits that are the Bryant soliton (the unique rotationally symmetric steady Ricci soliton) and the round cylinder $S^{2} \times \mathbb{R}$, depending on how the sequence of points and times about which one rescales are chosen. These are the only backward-in-time limits of Perelman's ancient solution. In [18] Perelman conjectured the following classification of $\kappa$-noncollapsed ancient solutions.

Conjecture. The only $x$-noncollapsed ancient solutions to the three-dimensional Ricci flow are the contracting spheres, Perelman's solution, the contracting round cylinders, and the unique rotationally symmetric steady Ricci soliton, known to be the Bryant soliton.

Aiming to resolve Perelman's conjecture, the authors and Hamilton initiated a research program to study the classification of ancient solutions to geometric flows under natural geometric conditions such as noncollapsedness introduced above, curvature bounds, or convexity properties in the extrinsic case. More recently, the authors have also collaborated with S. Angenent and S. Brendle.

In what follows we will describe some of the main classification results, starting from the heat equation on 
manifolds and ending with three-dimensional Ricci flow as described above.

Ancient solutions to the heat equation on manifolds. A well-known result by S. T. Yau dating back to 1975 asserts that on a complete noncompact Riemannian manifold $M^{n}$ of dimension $n \geq 2$ with nonnegative Ricci curvature, any positive harmonic function $u$ must be constant. This is the analogue of Liouville's theorem for harmonic functions on $\mathbb{R}^{n}$. A natural question is whether the analogue of Yau's theorem holds for positive ancient or eternal solutions of the heat equation

$$
u_{t}=\Delta u \quad \text { on } M^{n} \times(-\infty, T), \quad T \leq \infty,
$$

under the same assumptions on $M^{n}$. The answer to this question is negative, as it can be easily seen by the eternal solution to the heat equation on $M=\mathbb{R}^{1}$, given by $u(x, t)=e^{x+t}$. However, P. Souplet and Q. Zhang showed that the analogue of Yau's theorem holds under an extra growth assumption at infinity. Namely, if $u$ is a positive ancient solution to the heat equation on $M \times(-\infty, T)$ such that

$$
u(x, t)=e^{o(d(x)+\sqrt{|t|})} \quad \text { as } d(x) \rightarrow \infty,
$$

then $u$ is a constant. Also, if $u$ is any ancient solution to the heat equation such that

$$
u(x, t)=o(d(x)+\sqrt{|t|}) \quad \text { as } d(x) \rightarrow \infty,
$$

then $u$ is a constant. Note that this last estimate is also sharp in the spatial direction due to the example $u(x, t)=x$. The result of Souplet and Zhang is based on local gradient estimates for positive solutions (bounded or not) to the heat equation. Such estimates, first introduced by P. Li and Yau have played a fundamental role in the development of geometric flows.

Semilinear heat equation on $\mathbb{R}^{n}$. The simplest model of nonlinear diffusion is the semilinear diffusion equation

$$
u_{t}=\Delta u+|u|^{p-1} u
$$

for different exponents $p>1$. This equation is often seen as a prototype in the analysis of singularities of more complex geometric flows such as the mean curvature flow and the Ricci flow.

The nonlinearity in equation (1) with $p>1$ makes the solution blow up at a finite time $T<+\infty$. This can be easily observed by looking at the ODE $u^{\prime}(t)=|u|^{p-1} u$. If a solution $u$ of (1) has a finite time singularity at a point $(a, T)$, then

$w_{a}(y, \tau):=(T-t)^{\frac{1}{p-1}} u(x, t), y=\frac{x-a}{\sqrt{T-t}}, \tau=-\log (T-t)$,

satisfies equation (2). The study of $u$ near a blow-up point $(a, T)$ is equivalent to the study of the long-time behavior of the rescaled solution $w_{a}$. Given any sequence $\tau_{n} \rightarrow \infty$, one defines the solutions $w_{a}^{n}(x, \tau):=w_{a}\left(x, \tau+\tau_{n}\right)$, which are defined on $-\log T-\tau_{n} \leq t<+\infty$. Standard regularity theory for parabolic equations implies that, passing to a subsequence, the sequence $w_{a}^{n}$ converges, as $n \rightarrow \infty$, to an eternal solution $w$ of equation

$$
w_{\tau}=\Delta w-\frac{y}{2} \cdot \nabla w-\frac{w}{p-1}+w^{p}, \quad(y, \tau) \in \mathbb{R}^{n} \times \mathbb{R} .
$$

The study of the long-time behavior of $w_{a}$ is closely related to the classification of eternal solutions of (2).

In [17], F. Merle and H. Zaag provided the classification of positive bounded eternal solutions to equation (2) in the range of exponents $1<p<(n+2) /(n-2)$ following the previous well-known classification results by Y. Giga and R. Kohn. More precisely, in [17] it is shown that if $w$ is a nonnegative bounded eternal solution of equation (2), then $w$ is independent of the spatial variable $y$ and hence

$$
w=0 \text { or } w=\kappa \text { or } w(s)=\phi\left(s-s_{0}\right) \text { for some } s_{0} \in \mathbb{R}
$$

with $\phi(s)=\kappa\left(1+e^{s}\right)^{-\frac{1}{(p-1)}}$.

The significance of the Merle-Zaag theorem is the classification of the eternal solutions to (2) that connect the steady states $\lim _{\tau \rightarrow-\infty} w(y, \tau)=w_{-\infty}(y)=\kappa$ and $\lim _{\tau \rightarrow \infty} w(y, \tau)=w_{+\infty}(y)=0$. These solutions are shown to be independent of the spatial variable $y$, hence given by $\phi(s)$. Its proof is very involved and strongly relies on analyzing the behavior of the solution $w(y, \tau)$ near $\tau \rightarrow-\infty$, in terms of its projections on the positive, zero, and negative eigenspaces of the linearized operator $\mathcal{L} v:=\Delta v-$ $\frac{1}{2} y \cdot \nabla v+v$ at $w=\kappa$. Notice that in terms of $w:=v-\kappa$, equation (2) takes the form

$$
w_{\tau}=\mathcal{L} v+f(v)
$$

with superlinear error $f(v):=(v+\kappa)^{p}-\kappa^{p}-p \kappa^{p-1} v$. Equation (2), with different nonlinear error functions $f(v)$, often arises in the analysis of singularities in geometric flows, in particular in neck-pinches in the mean curvature flow and the Ricci flow.

Ancient compact solutions to curve shortening flow and Ricci flow on surfaces. The simplest geometric flow is the evolution of curves $\Gamma_{t} \subset \mathbb{R}^{2}$ embedded in the plane by the curve shortening flow. This is the evolution of an embedding $F(\cdot, t): M^{1} \rightarrow \mathbb{R}^{1}$ with $\Gamma_{t}:=F\left(M^{1}, t\right)$ by the equation

$$
\frac{\partial F}{\partial t}=-\kappa \nu,
$$

which moves each point $P=F(p, t) \in \Gamma_{t}$ in the direction of the inner normal vector $\nu$ to the curve at $P$ by a speed that is equal to the curvature $\kappa$ of the curve.

The curve shortening flow is well studied, and most questions regarding the existence of the flow and its asymptotic behavior have been well understood. M. Gage in 1984 and Gage and Hamilton in 1984 showed that the curve shortening flow shrinks closed convex curves embedded in $\mathbb{R}^{2}$ to points. In addition, the curve remains convex and becomes asymptotically circular close to its 
extinction time. This is the one-dimensional analogue of Huisken's result for mean curvature flow in [15]. However, M. Grayson showed that the curve shortening flow has a surprising and beautiful property not appearing in any other flow: if the initial data $\Gamma_{0}$ is any embedded closed curve in $\mathbb{R}^{2}$, then the solution $\Gamma_{t}$ of (4) does not develop any singularities before it becomes strictly convex; then it becomes circular as it shrinks to a point according to the Gage-Hamilton result. This is in contrast with the higherdimensional mean curvature flow, known for developing many exotic singularities that have been the subject of study in recent years.

As a first step to understanding ancient solutions to nonlinear geometric flows, the authors together with Hamilton established the classification of embedded ancient closed convex solutions $\Gamma_{t}$ of the curve shortening flow in $\mathbb{R}^{2}$, that is, solutions defined for all $t \in(-\infty, T)$, for some $T<+\infty$. By the Gage-Hamilton result discussed above, the family of curves $\Gamma_{t}$ will become circular as they become extinct to a point at time $T$.

Let $\theta$ be the angle between the tangent vector of $\Gamma_{t}$ and the $x$ axis. For convex curves we can use $\theta$ as a parameter and consider the evolution of the curvature $x(\theta, t)$ as a $2 \pi$-periodic function of the angle $\theta$ and of time $t$. It was computed by Gage-Hamilton that in this case $\kappa$ evolves by the quasilinear parabolic equation

$$
\kappa_{t}=\kappa^{2} \kappa_{\theta \theta}+\kappa^{3} .
$$

It turns out that for convex curves the evolution of the family $\Gamma_{t}$ is completely described by the evolution (5) of the curvature $x$.

The ancient solution to (4) defined by

$$
\kappa(\theta, t)=\frac{1}{\sqrt{2(T-t)}}
$$

corresponds to a family of contracting circles. This solution belongs to the category of contracting self-similar solutions (these are solutions of the flow whose shapes change homothetically during the evolution). However, Angenent discovered other compact ancient solutions to (4) that are not self-similar. We refer to them as Angenent ovals. They are given in closed form in terms of the curvature $\kappa(\theta, t)$ by

$$
\kappa^{2}(\theta, t)=\lambda\left(\frac{1}{1-e^{-2 \lambda(T-t)}}-\sin ^{2}(\theta+\gamma)\right)
$$

for two parameters $\lambda>0$ and $\gamma \in \mathbb{R}$. These solutions are not self-similar. It turns out that such ancient solutions are typical in other flows as well, in particular in the mean curvature flow and the Ricci flow in dimensions $n \geq 2$.

In [10] the authors jointly with Hamilton showed that the only ancient embedded closed convex solutions to the curve shortening flow in $\mathbb{R}^{2}$ are either the family of contracting circles or the Angenent ovals.
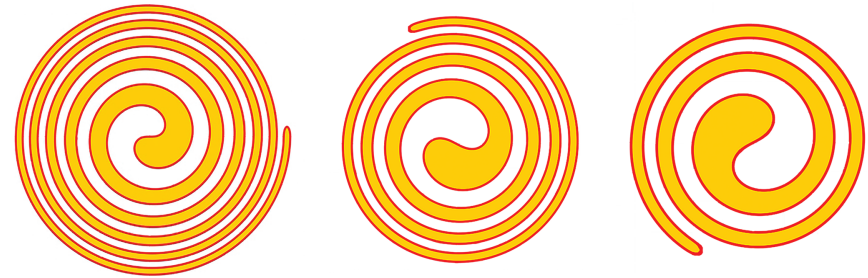

Figure 2. A nonconvex ancient solution to curve shortening flow at times $t_{1}<t_{2}<t_{3}$. Shots from a video created by Sigurd Angenent.

The proof of this result uses the explicit form of the Angenent ovals in an important way. It involves the monotonicity of a certain Lyapunov functional that vanishes on both the contracting circles and the Angenent ovals. By analyzing the behavior as $t \rightarrow-\infty$ and $t \rightarrow T$, one is able to show that this Lyapunov functional is identically equal to zero on any ancient convex solution. One then deduces the desired classification result. It is crucial for the proof that on an ancient convex solution the curvature $\kappa(\theta, t)$ is monotone in $t$. This follows by Hamilton's differential Harnack inequality, which implies that $\kappa_{t} \geq 0$.

It turns out that the convexity assumption is necessary in the classification result that was discussed above. In fact, it is broadly believed that many exotic nonconvex ancient solutions exist. Figure 2 shows shots from a computer simulation created by Angenent that describes the evolution in time of an ancient nonconvex solution to the curve shortening flow. The mathematical construction of this solution has recently been done in a work of Angenent in collaboration with Yongzhe Zhang, Ilyas Kahn, and Connor Olson.

It is broadly observed that the $n$-dimensional mean curvature flow has many features similar to the $n+$ 1)-dimensional Ricci flow. Since the curve shortening flow can be viewed as a one-dimensional mean curvature flow, one expects that the classification problem discussed above has an analogue in the two-dimensional Ricci flow. In fact this was shown by the authors and Hamilton in [11] The Ricci flow analogue of the Angenent ovals is the King solution, which was discovered, much earlier in 1993, by the applied mathematician J. R. King in the context of the logarithmic fast diffusion equation. Similarly to the result in [10] that was described above, it was shown in [11] that the only ancient compact solutions of the Ricci flow on $S^{2}$ are the contracting spheres and the King solution. The proof of this result also uses the explicit form of the King solution in an important way. However, it is much more complex. It combines several analytical and geometric arguments, such as monotonicity formulas, critical a priori estimates, blow-up arguments, the maximum principle, and an isoperimetric inequality. Let us remark that the King solution does not satisfy the $x$-noncollapsed property. It is, as we say, a collapsed solution. The result in [11] classifies all compact ancient solutions to the two-dimensional Ricci flow. In higher 
dimensions, the classification of collapsed ancient solutions is still widely open.

Ancient noncollapsed solutions to mean curvature flow. We have just discussed the classification of ancient solutions to curve shortening flow and Ricci flow on surfaces, and we saw that both results share some crucial similarities. One of them is the fact that all ancient solutions under consideration are given in explicit form. However, no such property holds in the three-dimensional Ricci flow, the original goal of our project. This property holds neither in its analogue, the two-dimensional mean curvature flow, nor, more generally, in mean curvature flow in any dimension $n \geq 2$, which will be discussed next.

We say that $M_{t}=F\left(M^{n}, t\right)$, where $F(\cdot, t): M^{n} \rightarrow \mathbb{R}^{n+1}$ is a solution to the mean curvature flow equation if

$$
\frac{\partial}{\partial t} F=-H \nu
$$

where $H$ is the mean curvature of $M_{t}$ and $\nu$ is the outward unit normal vector. While this flow reduces to a quasilinear parabolic equation, it can also be considered as the heat equation on the evolving manifold $M_{t}$, as the above equation can be rewritten in the form

$$
\frac{\partial}{\partial t} F(p, t)=\Delta_{M_{t}} F(p, t) .
$$

We have already seen that Huisken in [15] showed that if $M_{0} \subset \mathbb{R}^{n+1}$ is a closed convex embedded hypersurface, the mean curvature flow starting at $M_{0}$ converges to a round point. However, in contrast with the onedimensional case, in higher dimensions the convex case is very special. In more general situations, without the convexity assumption local singularities may likely occur. For example, if $M_{0}$ looks like a dumbbell, the neck pinches off; that is, a blow-up limit around a singularity is a round shrinking cylinder.

The maximum principle implies that the distance between two mean curvature flow solutions is nondecreasing in time. As a consequence, a comparison argument with shrinking spheres implies that every compact mean curvature flow develops singularities in finite time. It is known that at the first singular time $T$, the $\lim \sup _{t \rightarrow T} \sup _{M_{t}}|A|(\cdot, t)=$ $+\infty$. Huisken's well-known monotonicity formula shows that the integral of the $n$-dimensional backward heat kernel decreases in time. This monotonicity formula has played an essential role in singularity analysis until today.

In the context of mean curvature flow there are several notions of weak solutions, which enable one to continue the flow through singularities without performing surgery. Such notions of weak solutions are still missing in general in the context of Ricci flow. As we saw earlier, one of the ways to continue the flow past the singular time in both the Ricci flow and the mean curvature flow is the flow with surgery. This includes cutting the hypersurface along necks, gluing in caps, and continuing the flow of the pieces, while the components of known geometry and topology are discarded (see [16] and [9] for results of mean curvature flow with surgery in different settings). Furthermore, similar to the Ricci flow, the study of ancient solutions could potentially help with surgeries in more general settings. In fact, similar to Perelman's famous work on the Ricci flow [18], which shows that the high curvature regions are modeled on ancient solutions with nonnegative curvature and are $\kappa$-noncollapsed, results were obtained for mean curvature flow assuming mean convexity and embeddedness. One advantage of mean curvature flow with surgery is that the entire discussion takes place in the framework of smooth differential geometry, in contrast with other approaches of weak solutions.

In $[1,2]$, the authors and Angenent considered ancient compact solutions to the mean curvature flow in dimensions $n \geq 2$, i.e., solutions that are defined for $t \in(-\infty, T)$ for some $T<+\infty$.

There is a notion of $\alpha$-noncollapsed solutions to the mean convex MCF, which is the analogue to Perelman's $\kappa$-noncollapsing condition for the Ricci flow. This was first introduced by W. Sheng and X. J. Wang. The results that we will mention below hold in any dimension, but for simplicity we will focus only on the case of surfaces in $\mathbb{R}^{3}$.

In recent important work, Brendle and K. Choi (see [8] and references within) gave the complete classification of noncompact ancient solutions to mean curvature flow on surfaces that are strictly convex. More precisely, they showed that any noncompact and complete ancient solution to mean curvature flow that is strictly convex and noncollapsed is the Bowl soliton, up to scaling and ambient isometries. The Bowl soliton is the unique rotationally symmetric, strictly convex solution to mean curvature flow that translates with unit speed. It has the approximate shape of a paraboloid, and its mean curvature is largest at the tip.

In [1] and [2] we focused on ancient noncollapsed closed solutions to the mean curvature flow. Wang showed in this case that the backward limit as $t \rightarrow-\infty$ of the rescaled flow is either a sphere or a cylinder $\mathbb{R} \times S^{2}$ of radius $\sqrt{2}$.

It is known that if the backward limit is a sphere, then the ancient solution has to be a family of shrinking spheres. Hence, to classify ancient compact noncollapsed solutions one may restrict to the ones that are non-self-similar. We will refer to them as ancient ovals. Based on formal matched asymptotics, Angenent conjectured the existence of an ancient oval solution defined on $t \in(-\infty, T)$ for some $T<$ $+\infty$, which as $t \rightarrow-\infty$ becomes more and more oval in the sense that it looks like a round cylinder $\mathbb{R} \times S^{2}$ in the middle region and like a rotationally symmetric translating soliton (the Bowl soliton) near the tips. A variant of this conjecture had been shown by B. White in 2003, but 


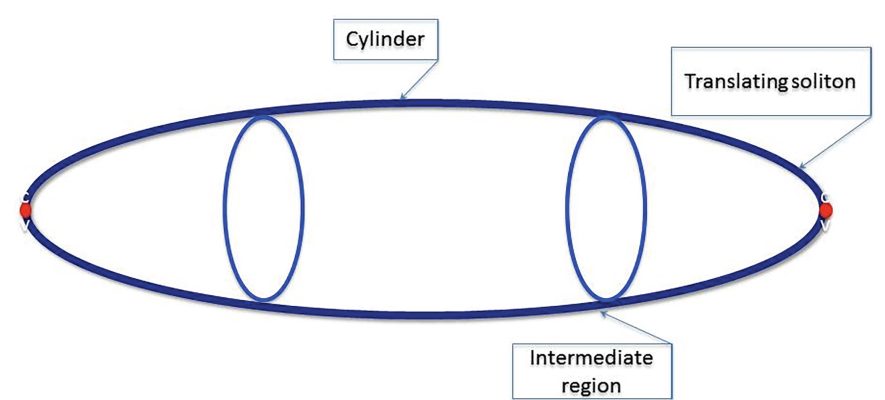

Figure 3. Behavior of ancient ovals and Perelman's solution as $t \rightarrow-\infty$, after scaling.

the approximate form of these solutions was not shown. More recently, R. Haslhofer and O. Hershkovits carried out White's construction in more detail, including, in particular, the study of the geometry at the tips. We will refer to them as White ancient ovals.

The main classification result of the authors with Angenent in [2] establishes the uniqueness of ancient ovals. More precisely, any ancient oval in $\mathbb{R}^{3}$ is equal to the White oval solution up to ambient isometries, scaling, and translations in time. A similar result was shown to hold in any dimension $n \geq 3$ (see [2] for the detailed statement). The proof has two steps: one first shows the rotational symmetry of such solutions, and then one establishes the uniqueness of rotationally symmetric ancient ovals. Analyzing the asymptotic behavior as $t \rightarrow-\infty$ of the rescaled ancient ovals plays an important role in our proof. This was done in [1], where the precise unique asymptotics were described in each of the three regions: the parabolic region, the intermediate region, and the tip region (see Figure 3).

The above result deals with noncollapsed solutions. A recent important work by T. Bourni, M. Langford, and G. Tinaglia [4] addresses the uniqueness of ancient collapsed mean curvature flow solutions under rotational symmetry. This work opens new directions in the classification of collapsed solutions.

The classification of $x$-solutions to three-dimensional Ricci flow. We will next discuss recent works by the authors and Brendle on the classification of noncollapsed solutions to the three-dimensional Ricci flow, which finally resolves the conjecture by Perelman, as stated at the beginning of this article.

We say that $(M, g(t))$ is a Ricci flow solution starting at the initial metric $g_{0}$ if it satisfies the equations

$$
\frac{\partial}{\partial t} g_{i j}=-2 R_{i j}, \quad g_{i j}(\cdot, 0)=g_{0 i j}(\cdot),
$$

where $R_{i j}$ is the Ricci curvature.

In a recent important work, Brendle [5] resolved the classification of ancient complete noncompact $\boldsymbol{k}$-noncollapsed solutions, showing that they are either the round cylinders or steady Ricci solitons. After providing the classification of those solutions under the assumption of rotational symmetry, he shows that any three-dimensional $\kappa$-noncollapsed noncompact ancient Ricci flow solution has to be rotationally symmetric.

Regarding the classification of closed $\kappa$-noncollapsed ancient solutions, Brendle in [6] has recently modified his arguments from [5] to show that such solutions must be rotationally symmetric. What remained for the resolution of Perelman's conjecture was the classification of rotationally symmetric closed solutions. It turned out that such a result could now be approached using the techniques that the authors have developed over the years, in particular the techniques from the mean curvature flow classification results in $[1,2]$.

Indeed, very recently in [12] the authors presented the proof of the uniqueness under the assumption of reflection symmetry. In a forthcoming joint work, the authors with Brendle are able to remove the reflection symmetry assumption from the result above, leading to the complete classification of closed ancient $x$-noncollapsed solutions to the threedimensional Ricci flow, as envisioned by Perelman.

Our uniqueness result relies heavily on analyzing the limits, as $t \rightarrow-\infty$, of any given solution. We show that such a limit either has to be a round sphere or has a round cylinder as one of its backward limits. In the latter case, in a joint work with Angenent [3], assuming reflection symmetry, the authors show that every three-dimensional $k$ solution that has the round cylinder as one of its backward limits has unique asymptotics. We describe the precise asymptotics in each of the three regions: the parabolic region, the intermediate region, and the tip region. The asymptotics are similar to those of two-dimensional mean curvature flow ovals (see Figure 3).

\section{Closing Remarks}

We have seen that the surgery procedure in both the mean curvature flow and the Ricci flow is important, since it allows us to continue the flow past singularities in a smooth framework. In the case of three-dimensional Ricci flow it has been very well understood due to works by Perelman and more recently by R. Bamler and B. Kleiner. The case of mean curvature flow is well understood in any dimension, under further assumptions on the initial surface.

In order to understand surgery in a more general setting in both flows one needs to fully capture all singularities and regions of high curvature. To achieve this, one needs to understand all limit flows, not only the tangent flows. Potentially, there may be very complex limit flows that are not solitons. Hence, a better understanding of ancient solutions is needed. The classification of ancient solutions in higher dimensions or under more general hypotheses is still out of reach, but the techniques described above could potentially lead to progress in that direction as well as to 
understanding ancient solutions and singularities in other nonlinear parabolic partial differential equations.

In previous classifications of ancient solutions to geometric flows, including those of the curve shortening flow [10], the two-dimensional Ricci flow [11], and the noncompact mean curvature flow $[7,8]$, an essential role was played by the fact that all such solutions were either given in closed form or were solitons (self-similar). One reason that the new techniques developed in $[1-3,12]$ are so significant is that they overcome such a requirement and potentially can be broadly used in various parabolic equations and, particularly, in geometric flows.

\section{References}

[1] Sigurd Angenent, Panagiota Daskalopoulos, and Natasa Sesum, Unique asymptotics of ancient convex mean curvature flow solutions. I. Differential Geom. 111 (2019), no. 3, 381455, DOI $10.4310 / \mathrm{jdg} / 1552442605$. MR3934596

[2] S. Angenent, P. Daskalopoulos, and N. Sesum, Uniqueness of two-convex closed ancient solutions to the mean curvature flow, arXiv: 1804.07230, 2018.

[3] S. Angenent, P. Daskalopoulos, and N. Sesum, Unique asymptotics of ancient compact non-collapsed solutions to the 3-dimensional Ricci flow, arXiv:1906.11967, 2019.

[4] T. Bourni, M. Langford, and G. Tinaglia, Collapsing ancient solutions of mean curvature flow, arXiv:1705.06981v2, 2017.

[5] S. Brendle, Ancient solutions to the Ricci flow in dimension 3, arXiv: 1811.02559

[6] S. Brendle, Rotational symmetry of ancient solutions to the Ricci flow in dimension 3 - the compact case, arXiv:1904.07835, 2019.

[7] Simon Brendle and Kyeongsu Choi, Uniqueness of convex ancient solutions to mean curvature flow in $\mathbb{R}^{3}$, Invent. Math. 217 (2019), no. 1, 35-76, DOI 10.1007/s00222-01900859-4. MR3958790

[8] S. Brendle and K. Choi, Uniqueness of convex ancient solutions to mean curvature flow in higher dimensions, arXiv 1804.00018

[9] Simon Brendle and Gerhard Huisken, Mean curvature flow with surgery of mean convex surfaces in $\mathbb{R}^{3}$, Invent. Math. 203 (2016), no. 2, 615-654, DOI 10.1007/s00222-015-0599-3 MR3455158

[10] Panagiota Daskalopoulos, Richard Hamilton, and Natasa Sesum, Classification of compact ancient solutions to the curve shortening flow, J. Differential Geom. 84 (2010), no. 3, 455-464. MR2669361

[11] Panagiota Daskalopoulos, Richard Hamilton, and Natasa Sesum, Classification of ancient compact solutions to the Ricci flow on surfaces, J. Differential Geom. 91 (2012), no. 2, 171-214. MR2971286

[12] P. Daskalopoulos and N. Sesum, Uniqueness of ancient compact non-collapsed solutions to the 3-dimensional Ricci flow, arXiv:1907.01928 2019.

[13] Richard S. Hamilton, Three-manifolds with positive Ricci curvature, J. Differential Geometry 17 (1982), no. 2, 255306. MR664497
[14] Richard S. Hamilton, The formation of singularities in the Ricci flow, Surveys in differential geometry, Vol. II (Cambridge, MA, 1993), Int. Press, Cambridge, MA, 1995, pp. 7-136. MR1375255

[15] Gerhard Huisken, Flow by mean curvature of convex surfaces into spheres, J. Differential Geom. 20 (1984), no. 1, 237-266. MR772132

[16] Gerhard Huisken and Carlo Sinestrari, Mean curvature flow with surgeries of two-convex hypersurfaces, Invent. Math. 175 (2009), no. 1, 137-221, DOI 10.1007/s00222-0080148-4 MR2461428

[17] Frank Merle and Hatem Zaag, Optimal estimates for blowup rate and behavior for nonlinear heat equations, Comm. Pure Appl. Math. 51 (1998), no. 2, 139-196, DOI 10.1002/(SICI)1097-0312(199802)51:2<139::AIDCPA2>3.0.CO;2-C. MR1488298

[18] G. Perelman, The entropy formula for the Ricci flow and its geometric applications, arXiv: math/0211159 2002.

[19] G. Perelman, Ricci flow with surgery on three-manifolds, arXiv:math/0303109 2003.

[20] G. Perelman, Finite extinction time for the solutions to the Ricci flow on certain three-manifolds, arXiv:math/0307245, 2003.

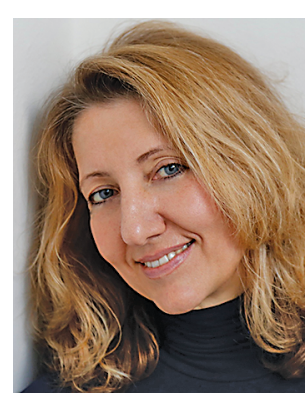

Panagiota

Daskalopoulos

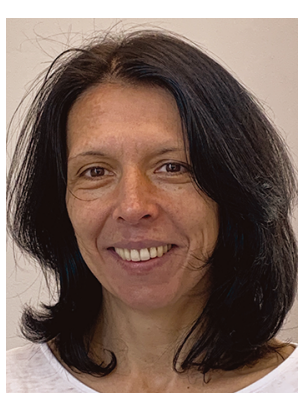

Natasa Sesum

\section{Credits}

Figure 1 is courtesy of Panagiota Daskalopoulos.

Figure 2 is by Sigurd Angenent.

Figure 3 is by Panagiota Daskalopoulos.

Photo of Panagiota Daskalopoulos is by Scott Mead.

Photo of Natasa Sesum is courtesy of Natasa Sesum. 